\title{
Bacterial spectrum of orofacial infections and their antibiotic resistance in Belarus
}

\author{
Corresponding author: \\ Arina Kabanova, PhD \\ Vitebsk State Madical University \\ Frunze Av., 27, 210023 Vitebsk, \\ Belarus \\ E-mail: arinakabanova@mail.ru
}

Medical Research Journal 2017; Volume 2, Number 4, 152-156 10.5603/MRJ.2017.0021 Copyright (C) 2017 Via Medica ISSN 2451-2591

\begin{abstract}
The purpose of the present study was to was to determine the pathogenic bacterial spectrum and bacterial antibiotic resistance in maxillofacial region of Belarussian patients.

Materials and methods. The study comprised inpatient who were treated by maxillofacial surgeons during 2014 in hospitals of Belarussian region: Brest, Mogilev, Vitebsk, Grodno and Gomel. It was a retrospective study, approval by the institution ethical committee. A microbiological sample was collected before antibiotic treatment. After meticulous disinfection of the skin around the planned extraoral incision site the aspiration of pus was attempted. If aspiration cannot be conducted, the samples were collected by swabbing the area after the incision and discharge of the pus. After sample collection, the material was immediately transported to the laboratory and further processed within 2 hours at the most. All specimens were then tested for the prevalence of the predominant bacteria and their antibiotic susceptibility Results. At the present stage in Belarussian hospitals the main agents isolating from inflammatory focus of the maxillofacial region are streptococci, staphylococci, enterobacteria, that corresponds with the global data. On the basis of the major pathogen antibiotic resistance data the following antibiotics for initial therapy until the receiving the results of bacteriological tests can be recommended: clindamycin (effective against all studied pathogenic species), ceftriaxone, fluoroquinolones (ciprofloxacin). Conclusions. It is necessary to conduct further systematic monitoring of microflora in patients with inflammatory diseases of maxillofacial area and studding the bacteria antibiotic resistance in order to improve the patient treatment efficiency.

Keywords: inflammation, maxillofacial area, microorganisms, Belarus
\end{abstract}

Med Res J 2017; 2 (4): 152-156

\section{Introduction}

Odontogenic inflammatory disease of maxillofacial area is an infectious and inflammatory process arising from the microflora spread through the tooth root canal affected by caries and its complications, or periodontal pocket in periapical tissue [1]. For a long time it was thought that the microbial landscape with odontogenic infection is represented mainly by monoculture of Streptococci or Staphylococci. According to the development of microorganism identification methods a lot of other microbial groups were studied and the role of gram-negative opportunistic pathogens was determined. Modern and advanced research technologies depend on the identification of genetic markers of microbial pathogens by polymerase chain reaction (PCR) [2].

Saprophytic microflora colonizing the mouth: Streptococcus mutans, Streptococcus milleri, Peptostrepto- coccus spp., Fusobacterium spp., Actinomyces spp. often appear as odontogenic infection agent. Nowadays more than 600 species of oral microorganisms were isolated and identified [3]. Microorganisms are usually localized on the oral cavity mucous membrane surface, in periodontal pockets, or decayed cavities [4]. According to the WHO classification the microflora of the oral cavity is mainly represented by anaerobic microbes growing in the dental plaque. Resident microflora of the oral cavity is extremely diverse, with low virulence properties [5] and includes bacteria, actinomycetes, fungi, protozoa, spirochetes, rickettsia, and viruses [6].

According to the contemporary professional literature in the field of odontogenic infectious-inflammatory process associations of 2-6 microorganism species had been identified, e.g. facultative anaerobes (Streptococcaceae and Staphylococcaceae) and obligate anaerobes (Bacteroides, Fusobacteriaceae. Peptostreptococcaceae) [7]. 
Different microorganisms may cause certain nosology. The development of odontogenic periostitis and osteomyelitis is caused by Staphylococcus aureus and Streptococcus spp., anaerobic flora (Peptococcus niger, Peptostreptococcus spp., Bacteroides spp.). Methicillin-sensitive Staphylococcus aureus (MSSA) is the main causative agent of nonodontogenic osteomyelitis in $52 \%$ cases, coagulase-negative staphylococci - $14 \%$, methicillin-resistant Staphylococcus aureus (MRSA) - $2 \%$, Pseudomonas aeruginosa - 4,4\%. Traumatic osteomyelitis is often caused by Staphylococcus aureus, Enterobacteriaceae spp., Pseudomonas aeruginosa [7].

According to European researchers, in patients with odontogenic infection, the most frequently isolated bacteria were as follows: anaerobes (8\%), gram-positive cocci (54\%), gram-negative cocci (8\%), Viridans group Streptococci (49 \%), non-haemolytic Streptococci (11\%), non-haemolytic Staphylococci (3\%), haemoIytic Streptococci $(0,5 \%)$, Enterecoccus spp. (1\%), Escherichia coli (1,5\%), Staphylococcus aureus (1\%), Bacteroides spp. (3\%) [8].

Thus, the causative agents of inflammatory diseases of maxillofacial area are represented by various species of microorganisms, the odontogenic infection is characterized as polyetiological. Due to the development of new microbiological methods, detection of the inflammatory disease etiology is expanding and updating constantly. All these facts indicate a need for further research in this direction.

Microbiological study of the maxillofacial inflammatory disease causative agents allows the identification of the pathogenic bacteria and determine their sensitivity to chemotherapeutic drugs, though ultimately providing optimum medical and surgical treatment tactics.

Therefore, the aim of the present study was to retrospectively analyse the pathogenic bacterial spectrum and bacterial antibiotic resistance in Belarussian patients with maxillofacial inflammatory disease.

\section{Material and methods}

\section{Study population}

The study comprised patients, who were treated by maxillofacial surgeons in 2014 in hospitals of Belarussian region: Brest, Mogilev, Vitebsk, Grodno and Gomel. Patients with maxillofacial inflammatory disease accounted for 35\% (238 people) of all patients hospitalized in the Maxillofacial Department in Brest, 38,5\% (628 people) - n Grodno, 28\% (727 people) - n Mogilev, 36\% (669 people) - n Vitebsk. A total of 2920 people with maxillofacial inflammatory disease were treated in 5 Belarussian regional hospital in 2014.
Inclusion criteria were odontogenic and nonodontogenic maxillofacial inflammatory process and no prior antibiotic treatment. Exclusion criterion was prior antibiotic treatment. All authors participating in the present study read the Declaration of Helsinki and the study was designed according to its guidelines. It was a retrospective study, approved by the local Ethics Committee.

\section{Data collection}

The skin and the oral mucosa of the infected site were rinsed with antiseptic in order to disinfect surface before incision. A microbiological sample was collected before antibiotic treatment. After meticulous disinfection of the skin around the planned extraoral incision site the aspiration of pus was attempted. If aspiration could not be conducted, the samples were collected by swabbing the area after the incision and discharge of the pus. After sample collection, the material was immediately transported to the laboratory and further processed within 2 hours at most. All specimens were then tested for the prevalence of the predominant bacteria and their antibiotic susceptibility [10-11]. The data was collected and analyzed in Microsoft Excell [12].

\section{Results}

\section{Isolated bacteria}

There were 65 isolates identified in the Department of Maxillofacial Surgery in Brest regional hospital. Staphylococci were isolated in most cases - 45 species $(70.0 \%)$, the most common group representative was Staphylococcus aureus. Enterobacteriaceae were presented by 7 strains (10.0\%). Among them Escherichia coli - 3 strains (4.0\%) and Klebsiella pneumoniae - 2 strains (3.0\%) were identified. Streptococcaceae were represented by Streptococcus pneumoniae - 11 strains (17.0\%). Nonfermentative gram-negative rods (NFGNR) were represented by Acinetobacter baummanii -2 strains (3.0\%).

There were 799 isolates identified in the Department of Maxillofacial Surgery in Mogilev regional hospital. Streptococci were isolated in most cases $(30.2 \%)$ and were represented by Streptococcus viridans - 204 strains (26.0\%), Streptococcus pneumoniae - 13 strains (1.6\%), Streptococcus pyogenes -21 strains (2.6\%). Among coagulase-negative Staphylococci (CNS) (43.0\%) there were Staphylococcus epidermidis - 270 strains (35.0\%) and Staphylococcus saprophyticus - 66 strains (8.0\%). Additionally, 139 strains (17.0\%) of Staphylococcus aureus were isolated. Enterococci (2.1\%) were represented by Enterococcus faecalis - 9 strains (1.0\%), Enterococcus 
faecium - 11 strains (1.1\%). Enterobacteriacae (2.72\%) were represented by Klebsiella spp. - 14 strains (1.7\%), Enterobacter spp.- 9 strains (1\%), Escherichia coli - 1 strain (0.02\%). Pseudomonas spp. were represented by $P$ seudomonas aeruginosa -6 strains (0.6\%).

There were 171 isolates identified in the Department of Maxillofacial Surgery in Gomel regional hospital. Streptococci were isolated in most cases (32.4\%), represented by Streptococcus viridans - 25 strains (14.0\%), Streptococcus pneumoniae - 3 strains (1.08\%), Streptococcus pyogenes - 10 strains (5.6\%). There were 50 strains (29.0\%) of Staphylococcus aureus. CNS (21.6\%) were identified as Staphylococcusepidermidis - 9 strains (5.0\%), Staphylococcus saprophyticus - 8 strains (4.6\%), Staphylococcus haemolyticus - 20 strains (12.0\%). Enterobacteriacae (8.9\%) were represented by Escherichia coli 7 (4.0\%), Escherichia cloacae - 3 (1.8\%), Citrobacter spp.- 1 (0.1\%), Klebsiella spp. -5 strains (3.0\%). Enterococcus spp. was revealed in $1.8 \%$ cases -3 strains. Members of the genus Pseudomonas were isolated in $3.0 \%$ of studies (5 strains). Other NFGNR were identified as Acinetobacter baummanii -1 strain $(0.1 \%)$.

There were 120 isolates identified in the Department of Maxillofacial Surgery in Vitebsk regional hospital. Staphylococci were isolated in most cases, among them Staphylococcus aureus - 18 strains (15.0\%), CNS - 76 strains (63.5\%). CNS were represented by Staphylococcus epidermidis. Streptococci were revealed in $8.3 \%$ of cases (10 strains). Enterobacteriacae (2.4\%) were identified as Escherichia coli - 1 strain $(0.8 \%)$ and Klebsiella pneumonia - 2 strains (1.6\%). Pseudomonas Aeruginosa was isolated in $5.0 \%$ of studies (6 strains), Acinetobacter spp. - n $08 \%$ (1 strain).

There were 162 isolates identified in the Department of Maxillofacial Surgery in Grodno regional hospital. Staphylococci were the most frequent bacteria detected - 139 strains (85.0\%), among them Staphylococcus aureus - 76 strains (47.0\%), CNS - 63 strains (38.0\%). CNS were represented by Staphylococcus epidermidis - 45 strains (28.0\%), Staphylococcus haemolyticus -12 strains $(6.5 \%)$, Staphylococcus hominis - 3 strains (2.0\%), Staphylococcus capitis -2 strains (1.0\%) and Stapylococcus warneri - 1 strain (0.5\%). Enterobacteriacae were represented by 4 strains (2.0\%), among them Klebsiella oxytoca - 1 strain $(0.5 \%)$, Klebsiella pneumoniae - 1 strain (0.5\%), Pseudomonas mirabilis - 2 strains (1.0\%). There were 11 strains $(7.0 \%)$ of Streptococcaceae and they were represented by Streptococcus pyogenes - 3 strains (2.0\%), Streptococcus mitis - 2 strains (1.0\%), Streptococcus sanguinis -2 strains (1.0\%), Streptococcus anginosus - 2 strains (1.0\%) and Streptococcus pneumoniae -2 strains (1.0\%). NFGNR were represented by Acinetobacter - 1 strain (0.5\%) and Pseudomonas aeruginosa - 1 strain (0.5\%). Additionally, 1 strain of Enterococcus spp. (0.5\%) was isolated.

\section{Antibiotic resistance}

Antibiotic resistance of Staphylococcus aureus was studied in Departments of Maxillofacial Surgery in Vitebsk, Brest, Gomel and Mogilev regional hospitals.

The greatest number of MRSA was detected in Brest $-27.8 \%$. In Vitebsk this rate was $14.8 \%$, in Mogilev - 10.0\%, and in Gomel - 5.6\%.

Staphylococcus aureus, isolated from the patients with maxillofacial inflammatory process in Vitebsk, was the least resistant to vancomycin $(0 \%)$, clindamycin $(0 \%)$, amikacin $(3.7 \%)$, cefazolin $(8.3 \%)$, ceftriaxone (11.5\%), lincomycin (13.0\%), gentamicin (14.8\%), ciprofloxacin (18.5\%), and ofloxacin (18.5\%). The highest level of resistance was noted for cefotaxime (25.0\%).

Staphylococcus aureus, isolated in Brest Department of Maxillofacial Surgery, was the least resistant to lomefloxacin, vancomycin, gentamicin, ofloxacin, trimethoprim, pefloxacin (for all 0\%), clindamycin (4.0\%), linezolid (8.3\%), tigecycline (16.7\%), and levofloxacin (20.0\%). The highest antibiotic resistance level of Staphylococcus aureus was revealed for erythromycin (71.4\%).

In the Department of Maxillofacial Surgery in Mogilev regional hospital Staphylococcus aureus showed the least resistance to vancomycin $(0 \%)$, clindamycin $(5.0 \%)$, gentamicin $(6.0 \%)$, levofloxacin $(6.0 \%)$, the highest resistance level was detected for amoxicillin (85.0\%).

In the Department of Maxillofacial Surgery in Grodno regional clinical hospital Staphylococcus aureus did not demonstrate the resistance to vancomycin, gentamycin, levofloxacin (for all 0\%). Resistance to linezolid was noted in $1.1 \%$ cases, clindamycine $-12.2 \%$, erythromycin $-17.8 \%$.

The resistance of Staphylococcus aureus to antibiotics differed in the studied Departments. The resistance of this pathogen to gentamycin was the lowest in Brest and Grodno (0\%), in Mogilev regional hospital it was $6.0 \%$ and in Vitebsk regional clinical hospital was the highest (14.8\%). Resistance of Staphylococcus aureus to clindamycin in Vitebsk was $0 \%$, Brest - 4.0\%, Mogilev - 5.0\%, Grodno - 12.2\%. Vancomycin resistance of Staphylococcus aureus was not revealed in any hospital.

Based on our findings concerning antibiotic sensitivity of Staphylococcus aureus in the Department of Maxillofacial Surgery of Brest, Vitebsk, Mogilev and Grodno regional clinical hospitals, clindamycin can be recommended as initial antibiotic therapy.

Gentamicin was effective against Staphylococcus aureus in Brest regional hospital and Grodno regional hospital. The sensitivity of Staphylococcus aureus to reserve antibiotic vancomycin was $100 \%$ in Vitebsk and 
Mogilev. The highest number of MRSA was identified in the Department of Maxillofacial Surgery in Brest regional hospital.

The highest number of methicillin-resistant strains of Staphylococcus epidermidis (MRSE) was detected in Grodno - 26.2\%, in Vitebsk - $22.4 \%$, in Mogilev $-4.0 \%$.

Staphylococcus epidermidis identified in the Department of Maxillofacial Surgery in Vitebsk regional clinical hospital was the least resistant to vancomycin $(0 \%)$, amikacin $(9.4 \%)$, ciprofloxacin $(11.5 \%)$, clindamycin $(14.0 \%)$, cefotaxime $(14.4 \%)$, ceftriaxone $(16.5 \%)$, cefazolin (16.6\%), ofloxacin (18.4\%), with the highest noted resistance to gentamicin (21.3\%), and lincomycin (28.8\%).

The least resistance of Staphylococcus epidermidis isolated in Grodno was to vancomycin, gentamicin and moxifloxacin (for all 0\%). Resistance to levofloxacin amounted to $4.8 \%$, to linezolid $-7.1 \%$, to clindamycin $-26.2 \%$.

Vancomycin resistance of Staphylococcus epidermidis identified in the Department of Maxillofacial Surgery in Mogilev regional hospital was $0 \%$, resistance to clindamycine was $6.0 \%$, to gentamycin $8.0 \%$, to levofloxacin $19.0 \%$.

The resistance of Staphylococcus epidermidis to vancomycin was not detected in the studied hospitals, the highest resistance to clindamicin was detected in Grodno, the highest resistance to gentamicin in Vitebsk.

In Gomel regional hospital Staphylococci antibiotic resistance accounted for vancomycin - 0\%, doxycycline $-3.3 \%$, ciprofloxacin $-6.6 \%$, clindamycine $-13.0 \%$, penicillin $-50.0 \%$. The number of methicillin-resistant staphylococci was $23,1 \%$.

Streptococci antibiotic resistance was identified in the Department of Maxillofacial Surgery in Vitebsk, Gomel, Brest.

Streptococcus spp. isolated in the Department of Maxillofacial Surgery in Vitebsk regional hospital had the least resistance to clindamycin and vancomycin (for all $0 \%)$, cefotaxime (4.0\%), ceftriaxone $(10.0 \%)$, ciprofloxacin (13.3\%), and doxycycline (20.0\%). The higher level of resistance was identified for co-trimoxazole (24.0\%), lincomycin (25.0\%), and ampicillin (28.6\%).

Streptococci identified in the Department of Maxillofacial Surgery in Gomel regional hospital had no resistance to ceftriaxone, levofloxacin, doxycycline, amoxicillin/clavulanic acid (for all $0 \%$ ). Resistance of Streptococci to clarithromycin amounted $3.4 \%$, to ofloxacin $-4.5 \%$, to clindamycin $-5.2 \%$, to ciprofloxacin $-5.6 \%$, to tetracycline $-11.1 \%$, to oxacillin $-15.4 \%$.

Streptococcus spp. identified in the Department of Maxillofacial Surgery in Brest regional hospital demonstrated no resistance to ceftriaxone, erythromycin, levofloxacin (for all $0 \%$ resistant strains). Resistance to lincomycin, oxacillin, tetracycline was $12.5 \%$, to erythromycin $-50.0 \%$.

Enterobacterial antibiotic resistance was identified in the Department of Maxillofacial Surgery in Vitebsk, Gomel, Grodno.

Enterobacteriaceae had no resistance to ceftazidime, cetriaxone, cefotaxime, imipenem, meropenem, amikacin, gentamicin, ciprofloxacin, cefoperazone/sulbactam in Vitebsk regional hospital. Resistance to amoxicillin/clavulanic acid amounted $50.0 \%$. The least resistance of Enterobacteriacea to imipenem (0\%), amikacin (0\%), ceftazidime (5.6\%), ciprofloxacin $(6.2 \%)$, ceftriaxone $(17.6 \%)$ was revealed in the Department of Maxillofacial Surgery in Gomel regional hospital. The highest resistance was detected for amoxicillin/clavulanic acid (31.2\%) and cefazolin (33.3\%). In Grodno regional hospital Enterobacteriacea had the least resistance to amikacin, ceftazidime, cefotaxime, cefepime, imipenem, meropenem, levofloxacin, tazobactam, tobramycin (0\%). Resistance to co-trimoxazole, ciprofloxacin and gentamicin was $9.1 \%$, to piperacillin - $11.0 \%$, to tetracycline, norfloxacin and amoxicillin/clavulanic acids $-25.0 \%$, to penicillin $-40.0 \%$.

\section{Discussion}

According to the modern researches the identified causative agents of maxillofacial inflammatory diseas are: Staphylococcus aureus - 26.7\%, Staphylococcus epidermidis - 17.8\%, Streptococcus spp. - 11.1\%, Escherichia coli and Proteus spp. $-11.1 \%$ of cases. In another study conducted in the Clinic of Oral and Maxillofacial Surgery in Nuremberg in 2010, Streptococci and Staphylococci were dominating among 40 identified aerobic bacterial cultures [13]. This data corresponds well with our results.

The study conducted by O. Ibragimov (2008) showed, that there are certain differences in the selected wound microflora in children with infectious maxillofacialinflammatory disease. In the microflora Staphylococcus aureus was dominating (54.1\%) and the share of Enterobacteriacae accounted for $37.8 \%$ of all isolated microorganisms. In addition, Candida albicans was recorded - 2.7\%; and even non-fermentative gram-negative rods (Pseudomonas aeruginosa) were determined $-5.4 \%$.

Therefore the results of microbiological testing of infectious maxillofacial inflammatory disease carried out in 5 Belarussian hospitals in 2014 were different. However, the general trend is that there is the main contribution of Streptococci and Staphylococci in the development of this disease.

According to the study of Staphylococci antibiotic resistance in the Department of Maxillofacial Surgery 
in Vitebsk, Gomel, Mogilev, Brest and Grodno regional hospitals, there was no resistance to vancomycin - the antibiotic of choice for treating MRSA. This result corresponds with the data about the absence of vancomycin-resistant Staphylococcus spp. in the Republic of Belarus. Clindamycin, doxycycline (Gomel), gentamicin (Brest, Grodno) can be recommended as empiric, first-line antibiotic therapy of inflammatory diseases of maxillofacial area. MRSA was isolated in all 5 hospitals. Identification of MRSA is associated with low effectiveness of beta-lactam antibiotics (penicillins, cephalosporins, carbapenems, combinations of penicillins with beta-lactamase inhibitors).

Effective antibiotics for streptococcal infections of the maxillofacial region are clindamycin, ceftriaxone and fluoroquinolones (levofloxacin, ciprofloxacin). Streptococcus spp. showed the greatest resistance to tetracyclines (tetracycline and doxycycline), that does not allow to recommend these antibiotics as an effective starting therapy.

The most effective antibiotics against Enterobacteriaceae are amikacin, ceftazidime, imipenem, ciprofloxacin, ceftriaxone, and the least effective - amoxicillin/clavulanic acid.

\section{Conclusions}

Maxillofacial inflammatory disease was a common cause of hospitalizations in the Department of Maxillofacial Surgery in Belarus in 2014. Bacteriological testing seems to be a standard of care in this group of patients. According to the results of bacteriological examinations, the highest number of clinical isolates were identified in the inflammatory focus of the maxillofacial area in Mogilev, a few less in Gomel, Vitebsk and Grodno regional clinical hospitals, the lowest number — $\mathrm{n}$ Brest regional hospital.

At the present stage in Belarussian hospitals the main agents isolated from the inflammatory focus of the maxillofacial region are Streptococci, Staphylococci, Enterobacteria, which corresponds well with the global data. According to our analysis of pathogens antibiotic resistance, the following antibiotics for initial therapy, until receiving the results of bacteriological tests, can be recommended: clindamycin (effective against all studied pathogenic species), ceftriaxone, fluoroquinolones (ciprofloxacin). It is necessary to conduct further systematic monitoring of microflora in patients with maxillofacial inflammatory disease and study the bacterial antibiotic resistance in order to improve the treatment efficacy.

\section{References}

1. Pinelis IS. Turchina YeV. Modern views on antibacterial therapy of pyo-inflammatory diseases of maxillofacial area. The Transbaikalian Medical Bulletin. 2014; 3: 182-188.

2. Tsarev VN. Nikolayeva YeN, Sarkisyan NA. Detection of periodontopathogenic bacterial markers in patients with infective endocarditis. Russian Dental Journal. 2009; 2: 32-34.

3. Keijser BJF, Zaura E, Huse SM, et al. Pyrosequencing analysis of the oral microflora of healthy adults. J Dent Res. 2008; 87(11): 1016-1020, doi: 10.1177/154405910808701104, indexed in Pubmed: 18946007

4. Bahl R, Sandhu S, Singh K, et al. Odontogenic infections: Microbiology and management. Contemp Clin Dent. 2014; 5(3): 307-311, doi: 10.4103/0976-237X.137921, indexed in Pubmed: 25191064.

5. Avila M, Ojcius DM, Yilmaz O. The oral microbiota: living with a permanent guest. DNA Cell Biol. 2009; 28(8): 405-411, doi: 10.1089/dna.2009.0874, indexed in Pubmed: 19485767

6. Timofeyev AA, Dakal AV, Kishkovskaya YN. Clinical flow of dontogenic inflammatory diseases of jaws and soft tissues for an ill with narcomania. Modern dentistry. 2009; 1: 94-98.

7. Kovaleva N, Zuzova AP. Pyoinflammatory Diseases Of The Maxillofacial Area: Aspects Of The Clinic, Microbiology, And Pharmacotherapy. Farmateka. 2011; 8: 34-38.

8. Götz C, Reinhart E, Wolff KD, et al. Oral soft tissue infections: causes, therapeutic approaches and microbiological spectrum with focus on antibiotic treatment. J Craniomaxillofac Surg. 2015; 43(9): 1849-1854, doi: 10.1016/j.jcms.2015.08.002, indexed in Pubmed: 26358301.

9. Skala LZ, Sidorenko SV, Nekhorosheva AG, et al. Practical aspects of modern clinical microbiology: Triada. ; 2004: 310.

10. Clinical and Laboratory Standard Institute. Performance Standards for Antimicrobial Susceptibility Testing, Nineteenth Informational Supplement M100-S18, Vol. 28, No. 1. Wayne, PA: CLSI. : 2008.

11. Poeschl PW, Crepaz V, Russmueller G, et al. Endodontic pathogens causing deep neck space infections: clinical impact of different sampling techniques and antibiotic susceptibility. J Endod. 2011; 37(9): 1201-1205, doi: 10.1016/j.joen.2011.05.029, indexed in Pubmed: 21846534

12. Rebrova OYu. Statistical analysis of medical data. The use of the application package STATISTICA: MediaSfera. ; 2002: 212.

13. Bogatov BB, Denis AG, Koliadov NF. [Rational antimicrobial therapy in patients with purulent inflammatory diseases of maxillofacial area]. Stomatologiia (Mosk). 2015; 94(1): 37-39, indexed in Pubmed: 25909613. 